\section{Dem Expertenurteil auf den Zahn gefühlt}

\author{
Gerade von der Biotechnologie mit ihrem hohen Innovationspotential in vielen \\ gesellschaftlichen Bereichen, wird ein Beitrag zu einer nachhaltigen Entwicklung \\ erwartet. Die Bewertung der Folgen dieser Technologie erfolgt jedoch unter hoher \\ Unsicherheit da Anwendungen immer noch in einer Frühphase sind. Für Entschei- \\ dungen trotz begrenztem Wissen kann die Unsicherheitsanalyse einen Weg weisen.
}

$\mathrm{D}$

Von Cornelia R. Karger ie Bio- und Gentechnologie ist eine Emerging Technology (1). Emerging Technologies sind in besonderem Maße wissenschaftsbasierte Innovationen, die hohe qualitative Sprünge in der Erkenntnis bedeuten können. Sie entwickeln sich dynamisch und befinden sich noch weitgehend im Vorfeld großräumiger technischer Anwendungen. Solche Innovationen bieten Chancen und bergen Risiken. Hohe Erwartungen sind mit einer hohen Unsicherheit in Bezug auf die Wirkungen dieser Technologien gepaart. Ob und welche der bio- und gentechnologischen Innovationen in Zukunft tatsächlich Marktreife erlangen werden, ist unsicher. Besondere Unsicherheit besteht allerdings darin, inwieweit die erhofften positiven Effekte tatsächlich eintreten. Dies betrifft die ökologischen Effekte ebenso wie die sozio-ökonomischen Auswirkungen der solchen Technologie. Zusätzlich besteht Unsicherheit bezüglich der Risiken. Die Bewertung einer Emerging Technology muss deswegen unter Unsicherheit erfolgen, insbesondere da die Beurteilungsunsicherheit häufig mit kontroversen wissenschaftlichen Auffassungen über mögliche Wirkungen der Technologie gepaart ist.

\section{Der Ansatz Unsicherheitsanalyse}

Gesellschaftliche Entscheidungen müssen trotz bestehender Unsicherheiten gefällt werden. Eine Vertagung des Problems - bis die Wissenschaft die offenen Fragen geklärt hat - ist bei Entscheidungen über Innovationsstrategien keine geeignete Lösung. Weichenstellungen müssen unter den Bedingungen des Wissens unter Unvollständigkeit und Ungewissheit getroffen werden (2). Allerdings öffnen Abschätzungsunsicherheiten den Raum für unterschiedliche Bewertungen als Ausgangspunkt für gesellschaftliche Konflikte. Die Unsicherheitsanalyse stellt daher die Charakterisierung des Wissens als Leitidee zur Bewertung der soll gewährleisten, die gesamte Bandbreite von Ar$\mathrm{gumenten} \quad \mathrm{zu} \quad$ e rfass e $\mathrm{n}$. Ausgangsbasis für die Unsicherheitsanalyse ist die Entwicklung eines Chancen- und Risikomodells. Dieses Modell bildet die potentiellen Chancen und Risiken anhand von Ursache-Wirkungsbeziehungen ab. Expertenurteile nehmen auf die im Modell spezifizierten Ursache-Wirkungsbeziehungen Bezug. Um die Wissensbasis und die Urteilsqualität der Experten zu eruieren, werden Begründungen in zweifacher Hinsicht eingefordert: für das Chancen- bzw. Risikourteil „Was spricht dafür/dagegen " und für die Urteils(un) sicherheit „Was macht mich sicher/unsicher" (siehe Abb.1).

Schließlich werden typische Argumente der Chancen- und Risikodebatte von den Experten im Hinblick auf ihre Zulässigkeit geprüft und ihre Relevanz für die Beurteilung von Chancen beziehungsweise Risiken eingeschätzt.

\section{Beispiel Grüne Gentechnik}

Die Grüne Gentechnik ist ein typisches Beispiel für eine Emerging Technology. Transgene Pflanzen sollen vor allem zu Ertragssteigerungen und effizienteren Anbaumethoden von Nahrungs- und Futterpflanzen mit weniger Chemieeinsatz sowie zu einem nachhaltigeren Umgang mit Energie und Rohstoffen fuihren. Insbesondere diejenigen Innovationen, in die hohe Erwartungen im Hinblick auf ihren Beitrag zu einer nachhaltigen Entwicklung gesetzt werden, wie zum Beispiel eine verbesserte Photosyntheseleistung, stehen aber noch am Anfang ihrer Entwicklung. Bei der Abschätzung der Folgen, vor allem der zweiten und dritten Generation der grünen Gentechnik, fehlen empirische Daten. Aber auch bei marktreifen Anwendungen, bei denen erste Daten schiedlicher Werthaltungen in fokussierte Einzelinterviews eingebunden. Der Expertenpluralismus

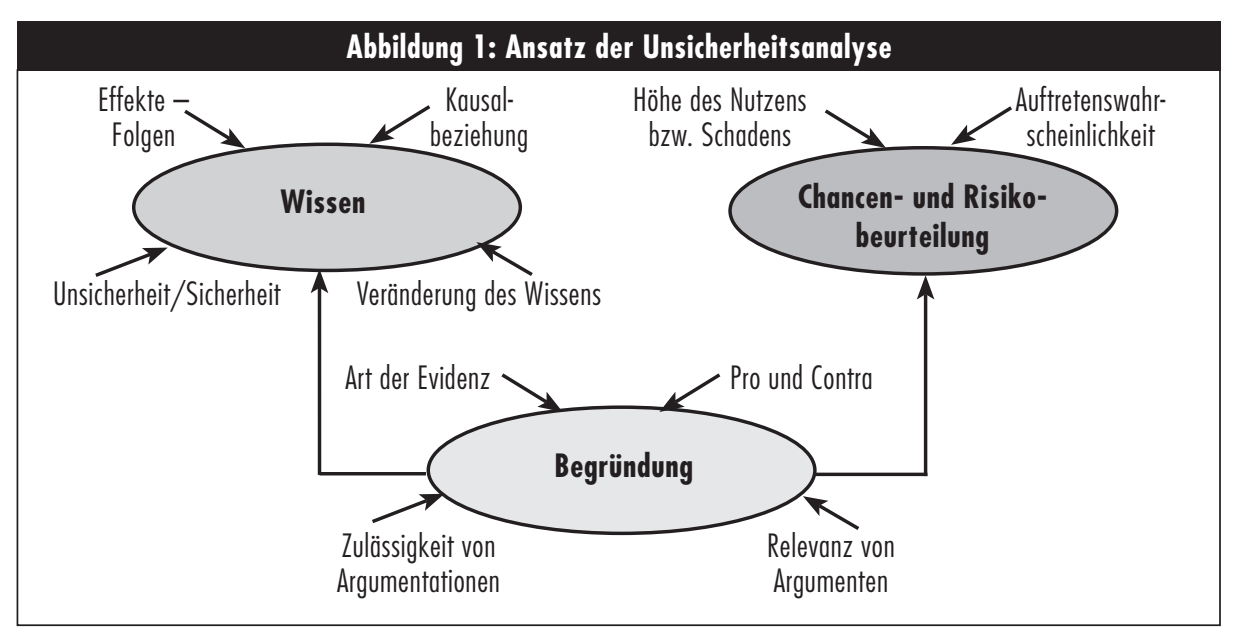

Quelle: Eigendarstellung 


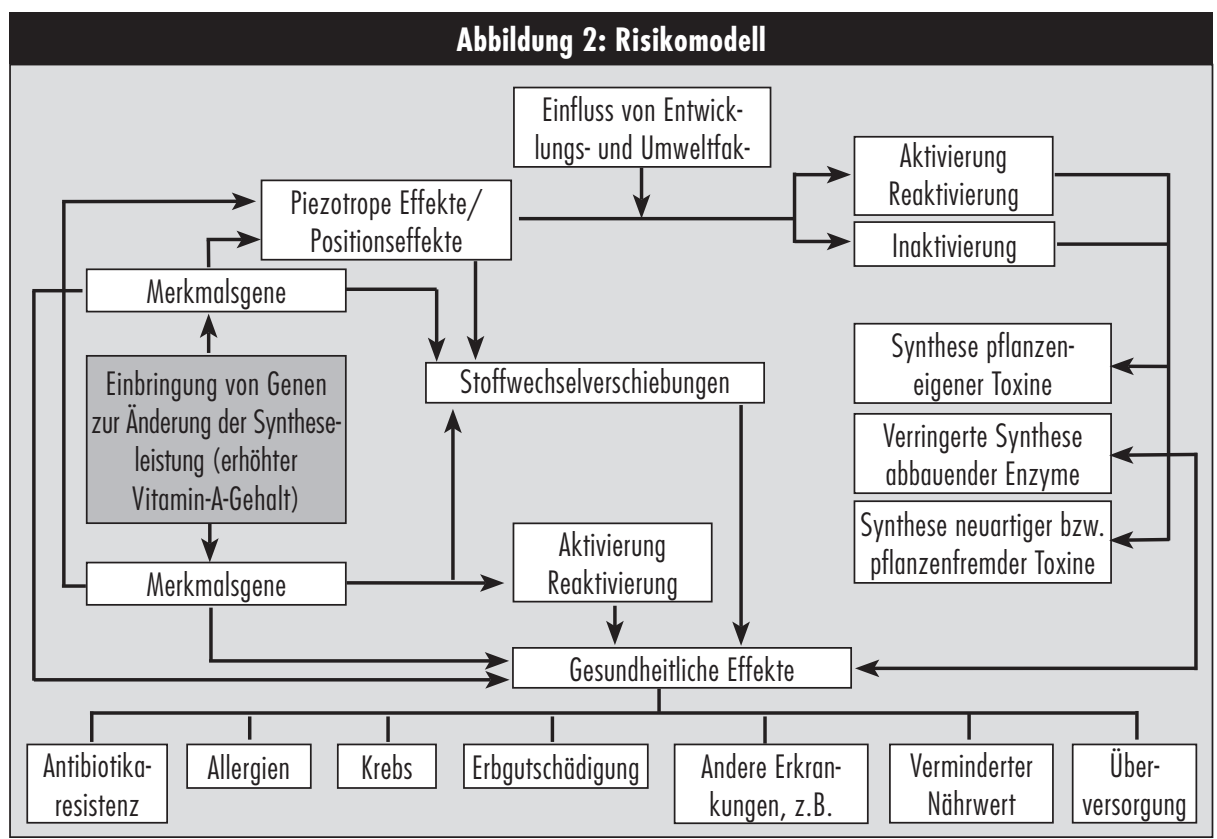

Quelle: Eigendarstellung

vorhanden sind, bleiben Unsicherheiten (4). Gleichzeitig wird eine Kontroverse um potentielle Umweltund Gesundheitsrisiken geführt.

In Interviews mit Experten wurde ein Risikomodell für das Beispiel des Einbringens von Genen zur Änderung der Syntheseleistung erarbeitet und für konkrete Anwendungsbeispiele, zum Beispiel Vitamin-A-Reis, spezifiziert (siehe Abb.2). Im Zentrum der Risikodebatte stehen hier vor allem potentielle unerkannte Stoffwechselverschiebungen als Ursache für adverse Effekte. Auf das Risikomodell bezogen sich Aussagen zu Wissen, Risikourteile, deren Begründungen sowie Urteilssicherheiten und -unsicherheiten.

\section{Experten überschätzen sich}

Die Ergebnisse zeigen, dass - wie erwartet - Experten sich in ihren Risikourteilen unterscheiden. Jedoch kommen Experten unterschiedlicher Werthaltungen nicht nur zu unterschiedlichen Ergebnissen, sie sind sich der Ergebnisse auch jeweils sicher. Es handelt sich also um eine Polarisierung in den Risikourteilen gepaart mit der Sicherheit, dass die jeweilige Einschätzung auch zutrifft. Die abweichenden Urteile können jedoch nicht gleichzeitig gültig sein. Daher kann das große Vertrauen der Experten in ihr eigenes Urteil als ein Hinweis auf overconfidence, also eine Überschätzung der Güte des eigenen Urteils interpretiert werden (5).

Darüber hinaus zeigt die Auswertung der subjektiven Urteilssicherheit, dass sich die Einschätzung der gesamten Gesundheitsschäden von denen der konkreten unterscheidet. Bei letzteren geben die Experten nicht nur sichere Urteile ab, sondern auch unsichere. Dies gilt unabhängig davon, ob das Gesamturteil pro oder contra Risiko abgegeben wurde. Offenbar werden bei der Angabe der Gesamtsicherheit die schwächeren Einzelevidenzen nicht in der Weise berücksichtigt wie die stärkeren, denn sonst müsste die Gesamturteilssicherheit geringer ausfallen. Inhaltlich ist eine unterschiedliche Gewichtung der Einzelevidenzen nicht begründet. Unsicherheiten bestehen nicht systematisch bei ,geringeren“ Gesundheitsschäden. Ein Gesamturteil „,sehr sicher“ bei unsicheren Einzelurteilen kann nur zustande kommen, indem letztere ,,ausgeblendet“ werden. Das subjektiv sichere Gesamturteil über den möglichen Schaden ist kein aggregiertes Urteil von Bewertungen einzelner Schäden. Es basiert vielmehr auf der Auswahl eines Einzelurteils. Befunde der kognitionspsychologischen Forschung zum Urteilen und Entscheiden belegen diese Interpretation. Bei der Bildung von Urteilen unter Unsicherheit setzen Menschen Heuristiken ein. So werden beispielsweise Informationen aus der Beurteilungssituation als Anker verwendet, von denen Schätzwerte für Wahrscheinlichkeiten nach oben oder unten adjustiert werden (5).

Die Urteilsbegründungen der Experten zeigen außerdem, dass Erwartungshaltungen eine wesentliche Rolle für die Beurteilung des Risikos spielen. Diejenigen, die kontra Risiko argumentierten, rekurrieren beispielsweise bei der Beurteilung von Gesundheitsrisiken aufgrund von pleiotropen Effekten/Positionseffekten auf Vertrauen in zukünftiges Wissen (siehe Abb.2): „Durch die Genomforschung werden wir eine noch bessere Abschätzung erreichen“, lautet etwa eine Aussage. Hingegen begründen diejenigen, die pro Risiko argumentierten, ihr Urteil mit den Grenzen der Wissenschaft: „Es wäre hilfreich, herauszufinden, wo die Gene inserieren. Dies setzt eine genaue Kenntnis des Genoms voraus. Ich bezweifle, ob man das wirklich kann“. Ebenso stehen sich in den Begründungen Erwartungshaltungen beispielsweise in Bezug auf das Risikomanagement gegenüber. Stützen sich Risikourteile auf Vertrauen, wird argumentiert, dass im Zulassungsverfahren Verfahren vorgeschrieben seien, die aufdecken, ob solche Stoffwechselverschiebungen aufgetreten sind. Werden Urteile mit Misstrauen in das Risikomanagement begründet, wird gegenteilig argumentiert, beispielsweise dass hier die methodische Sorgfalt fehle. Risikobeurteilungen basieren also einerseits auf Wissen und andererseits auf Erwartungs- und Werthaltungen.

Die Ergebnisse verdeutlichen die Notwendigkeit einer transparenten Beurteilungsgrundlage. Die Unsicherheitsanalyse ist geeignet, eine solche Grundlage zu schaffen. Unsicherheiten und implizite Erwartungshaltungen müssen in der wissenschaftlichen, aber auch gesellschaftlichen Diskussion um die Chancen und Risiken einer $E r$ merging Technology reflektiert werden.

\section{Anmerkungen}

(1) Day, G./ Schoemaker, P.J.H./ Gunther, R. E. ((Eds.): A Different Game. In: Wharton on Managing Emerging Technologies. New York 2000. S. 1-24.

(2) Funtowitz, S./ O'Connor, M./ Ravetz, J.: Challenges in the utilisation of science for sustainability. In: M. Catizzone (ed.): From Ecosystem Research to Sustainable Development. European Commission, Ecosystems Report № 26, Brussels 1999

(3) Courtney, H./ Kirkland, J./ Viguerie, P. : Strategy Under Uncertainty. In: Harvard Business Review on Managing Uncertainty, Cambridge 1999. S. 1-33.

(4) Karger, C.R./ Grutsch, M./ W-D. Fugger: Biotechnologie: Innovation und nachhaltige Entwicklung. In: A. Grunwald / R. Coenen (Hrsg.): Nachhaltigkeitsprobleme in Deutschland. Analyse und Lösungsstrategien. Berlin 2003. (5) Tversky, A./ Kahneman, D. : Judgment under uncertainty: Heuristics and biases. In: Science, 185, 1974. S. 1124 1131.

\section{Die Autorin}

Dipl. Psychologin Cornelia R. Karger ist wissenschaftliche Mitarbeiterin in der Programmgruppe Mensch, Umwelt, Technik (MUT) des Forschungszentrums Jülich.

Kontakt: MUT, Forschungszentrum Jülich, 52425 Jülich. Tel. 02461-612794, E-Mail: c.karger@fz-juelich.de 
(c) 20I0 Authors; licensee IÖW and oekom verlag. This is an article distributed under the terms of the Creative Commons Attribution Non-Commercial No Derivates License (http://creativecommons.org/licenses/by-nc-nd/3.o/), which permits unrestricted use, distribution, and reproduction in any medium, provided the original work is properly cited. 